\title{
Métaphore, poiétique et pensée scientifique
}

Jean-Jacques Wunenburger

\section{OpenEdition}

1 Journals

Édition électronique

URL : http://journals.openedition.org/ress/707

DOI : $10.4000 /$ ress.707

ISSN : 1663-4446

Éditeur

Librairie Droz

\section{Édition imprimée}

Date de publication : 1 février 2000

Pagination : $35-47$

ISBN : 2-600-00409-2

ISSN : 0048-8046

Référence électronique

Jean-Jacques Wunenburger, « Métaphore, poiétique et pensée scientifique », Revue européenne des sciences sociales [En ligne], XXXVIII-117 | 2000, mis en ligne le 17 décembre 2009, consulté le 19 avril 2019. URL : http://journals.openedition.org/ress/707 ; DOI : 10.4000/ress.707 


\title{
MÉTAPHORE, POIÉTIQUE ET PENSÉE SCIENTIFIQUE
}

\author{
L'usage de la métaphore n'est pas en soi un mal, et si, \\ comme je le crois, toute connaissance est métaphorique, \\ il est sans doute inévitable
}

La rationalisation du réel, tâche par excellence confiée à la scientificité, s'est vue longtemps assimilée au seul travail de conceptualisation et donc d'abstraction. L'épistémologie de la science a ainsi été préparée par la théorie philosophique de la connaissance, qui depuis Platon a déterminé et encensé les vertus du concept abstrait dans le processus d'intellection. Platon, le premier, en opposant connaissance sensible (doxa) et connaissance conceptuelle (dianoia), qui monte par degrés vers la représentation des Formes-essences des êtres physiques, a ainsi fait une place à la pensée pure. Ce besoin très cathartique de séparation de modes de pensée se justifiait d'autant plus que la philosophie naissante restait solidaire d'une langue encore très concrète, très imagée qui avait fait la fortune des premiers penseurs anté-socratiques ${ }^{2}$. Pourtant Platon lui-même connaît et exploite les ressources de la pensée symbolique et mythique, montrant d'ailleurs par là qu'on peut à la fois poursuivre une purgation de la pensée dans le sens de l'abstraction et réserver une place à une expression plus poétique.

Le cas de Platon, à l'égal d'ailleurs de celui d'Aristote dans sa Poétique et sa Rhétorique, est exemplaire et la leçon antique vaut peut-être toujours pour l'épistémologie contemporaine. Car si l'idéal d'abstraction s'est imposé à la rationalité scientifique, les ressources de l'image n'ont jamais cessées d'être reconnues, voire vantées. Plus même, la crise générale de la rationalité, typique de l'ère postmoderne, a favorisé dans beaucoup de secteurs la réévaluation de la pensée analogique $^{3}$. L'image n'est plus aussi autoritairement condamnée, elle connaît même une sorte de réhabilitation, quand elle ne se trouve pas, dans certains courants d'anarchisme méthodologique, mise en avant comme langage heuristique par excellence des sciences. Interroger les pouvoirs cognitifs de la métaphore est donc à la fois revenir à un problème inaugural de la philosophie occidentale, qui s'est posé à la naissance grecque de la raison, quand l'espace entre les mots et les choses restait encore saturé de poétique, et recentrer les débats de l'épistémologie

R. Thom, Apologie du logos, Hachette, 1990, p. 641.

2 Sur la langue philosophique des origines, voir $\mathrm{Cl}$. Ramnoux, Héraclite, entre les mots et les choses, Les Belles-Lettres, 1959.

3 Voir M. Cazenave (dir.), Sciences et symboles, Albin Michel, 1986; ou la position radicale de P. Feyerabend, Contre la méthode, Seuil, Points, 1989. 
des sciences et des sciences humaines en particulier, sur une question vive, celle des limites de la conceptualisation, envisagée comme procédure homogène, identitaire, autonome. L'enjeu de la question est non seulement de savoir si la rationalité ne doit pas être reconnue comme composite, plurielle, étagée, croisée, mais aussi de se demander si, en fin de compte, l'idéal de rationalité classique, celui des idées claires et distinctes, du sens propre, du concept défini, n'est pas une fiction, une utopie, en tout cas une Idée régulatrice plus qu'une réalité cognitive de fait.

\section{1. - LA MÉTAPHORE FAIBLE}

Une première approche du problème engage la question générale des rapports entre pensée abstraite, en philosophie ou dans les sciences, et rhétorique. Depuis l'Antiquité, en effet, il est admis qu'il faut non seulement valider des contenus de pensée vraie mais aussi, du fait de l'instrumentalisation sociale du discours, trouver des modes de déploiement efficaces, adaptés, pour convaincre ou persuader. Il ne suffit donc pas de formuler des idées selon leur seule nécessité logique, mais il faut encore savoir les présenter de telle sorte qu'elles recueillent l'assentiment de ceux à qui elles sont destinées à des fins de communication ou de conversion de croyance ${ }^{4}$. Mais les règles sélectionnées par les rhéteurs, destinées surtout à ceux dont la réussite communicationnelle est vitale (orateurs politiques, avocats, etc.) sont très vite apparues aussi comme des méthodes d'invention de discours, la rhétorique devant servir à bien organiser le développement d'une pensée, par la composition d'agencements ordonnés 5 .

Le succès voire l'abus de la rhétorique, périodiquement dénoncé dès l'Antiquité, a conduit souvent à des réactions virulentes destinées à dissocier méthode de bien penser et art de bien parler. Cette attitude engage d'ailleurs insensiblement une thèse logico-métaphysique, selon laquelle l'ordre des idées n'est pas totalement conditionné par l'ordre des mots, que pensée et langage, logique et grammaire relèvent de deux ordres séparés, et qu'en fin de compte la logique peut permettre de déterminer des contraintes propres à la formulation du vrai qui peuvent être déduites des seules catégories et relations abstraites de la pensée. Dans ce contexte le recours à l'image, à la comparaison et à la métaphore, se voit sévèrement réprimé, en tout cas contrôlé, surveillé, afin de mettre la spéculation abstraite à l'abri des séductions et de l'imprécision des jeux de langage. Cette économie malthusienne, rigoriste est favorisée par deux présupposés: le premier selon lequel la métaphore constitue toujours un déplacement d'une signification première, et donc un écart générateur de perte de précision, le second selon lequel, en conséquence, l'image ne peut être admise qu'à titre subsidiaire, afin de faciliter la divulgation de la pensée. Cette double orientation doit être explicitée, car elle constitue le substrat épistémique d'une certaine défiance à l'égard de la pensée analogique.

En effet, depuis Aristote, la métaphore est bien définie, conformément à son étymologie, comme un déplacement d'un sens propre, bref comme un «trope»

Sur cette naissance de la rhétorique, voir O. Reboul, Introduction à la rhétorique, PUF, 1991.

D'où les quatre fonctions traditionnelles de la rhétorique: l'invention (heurèsis), la disposition (taxis), l'élocution (lexis) et l'action (hypocrisis). 
fondé sur la perception de ressemblances: «La métaphore est le transport à une chose d'un nom qui en désigne un autre, transport ou du genre à l'espèce, ou de l'espèce au genre ou de l'espèce à l'espèce ou d'après le rapport d'analogie.» ${ }^{6} \mathrm{Le}$ langage étant assimilé à la science du mot propre, juste, à la nomination et à la dénomination adéquates, la comparaison et la métaphore, peu distinguées l'une de l'autre, se voient traitées comme des analogies qui instaurent des ressemblances entre des réalités à première vue dissemblantes. Dire qu'Achille est un lion, c'està-dire qu'il est courageux «comme» un lion, n'est pas gagner, mais plutôt perdre en rigueur et précision par rapport à l'attribution de la vertu du courage, qui porte sur une idée pleinement déterminée. La comparaison d'un homme et d'un lion ne saurait donc être qu'un détour que la pensée rationnelle devrait éviter. Une telle perspective s'intègre dans une philosophie du langage parfaitement cohérente, même si elle peut apparaître dogmatique et réductrice, et dont on peut énoncer quelques principes: le sens propre précède toujours le sens figuré, il lui est toujours supérieur en valeur, le sens univoque vaut mieux que le sens équivoque, la dénotation qui renvoie au référent objectif est plus informative que la connotation qui libère des associations subjectives, etc.

Corollairement, une telle approche conceptuelle, définitionnelle, identitaire va réserver la métaphore à une fonction secondaire, qui ne saurait être recommandée qu'une fois la pensée formée et énoncée selon les exigences de la justesse univoque. La métaphore s'apparente dans ce cas à la catégorie générale de l'allégorie, qui dans l'Antiquité servait à désigner toutes les procédures d'amplification imagée des contenus de pensée, au prix d'un recouvrement, d'une occultation du sens primitif, mais avec la satisfaction de produire des effets facilitant la récep$\operatorname{tion}^{7}$. Plus tard, l'allégorie se distinguera plus strictement du symbole, en ce qu'en elle l'image, narrative ou visuelle, dans le cas de la peinture, sert avant tout à rendre sensible (au sens de l'hypotypose de la rhétorique) un contenu idéel abstrait (l'idée de justice incarnée par l'image d'une femme tenant une balance). Parallèlement la métaphore se voit ainsi assigner une fonction illustrative d'un contenu préalablement pensé et énoncé dans sa substantialité idéelle propre. Ce «supplément» ou ce détour métaphorique a donc une finalité avant tout expressive, un sens préalablement arrêté et maîtrisé par la pensée et le langage, gagnant simplement en visibilité, en sensibilisation (la Versinnlichung, au sens de Kant). Bref, la métaphore est sauvée au titre de l'ornementation plaisante, de la didactique habile ou de la volonté esthétique de dramatisation et de théâtralisation des idées. La légitimation orthodoxe des images langagières mêle donc des motivations hétérogènes, ludiques, pédagogiques, rhétoriques, qui améliorent l'échange des pensées, dans certaines situations, mais ne contribuent jamais à la formation et à la formulation de ces mêmes pensées.

La conséquence d'une telle option méthodologique et épistémologique est décisive: la rationalité est censée constituer, depuis Descartes, une sphère cogni-

6 Aristote, Poétique. Au sens strict la métaphore, fondée sur la ressemblance, doit être distinguée de nos jours de la métonymie, fondée sur la connexion, et la synecdoque, fondée sur une correspondance. Mais épistémologiquement, la métaphore tend à désigner souvent l'ensemble de ces images, qui définissent les tropes.

7 J. Pépin, Mythe et allégorie, Aubier, 1958; G. Gusdorf, Les origines de l'herméneutique, Payot, 1988 . 
tive pure. Elle comprend des concept, jugement et raisonnement abstraits, c'est-àdire émancipés des données empiriques, des résonances affectives, des informations particulières tenant aux conditions spatio-temporelles accidentelles, etc. Platon déjà avait livré un modèle d'une telle herméneutique, dans le Phédon (qui a souvent été pris à tort pour le bréviaire du platonisme, alors qu'il ne modélise qu'un type de pensée anagogique réglée sur les mathématiques, qui implique le retrait hors du sensible), où l'on voit effectivement la pensée se détourner des déterminations empiriques pour développer un savoir à partir des seules Idées pures. Plus largement, la pensée scientifique moderne va mettre en œuvre ce programme en privilégiant dans l'intellection du réel, des quantifications et de purs modèles conceptuels, formels, algorithmiques. La mathématisation du réel pourra même autoriser la disqualification du langage ordinaire, suspecté de retenir dans ses mailles des lexiques ou des tournures pré-scientifiques, de sorte qu'un graphe par exemple sera toujours préféré à une image verbale.

C'est bien un tel rigorisme du constructivisme abstrait qui est développé par Gaston Bachelard. Certes ses travaux de psychologie de la connaissance et d'histoire de la formation des concepts scientifiques l'ont convaincu que notre esprit était spontanément habité par des images puissantes, résistantes, surdéterminées et surchargées d'affects inconscients, au point que l'imagination précède même toute perception. Mais si ces strates d'images peuvent nourrir la rêverie et devenir un foyer de créativité poétique, elles constituent par contre un obstacle épistémologique pour l'intelligence scientifique et doivent être soumises à des rectifications continues, même à une sorte de psychanalyse scientifique ${ }^{8}$. Pour Bachelard, la conceptualisation se développe donc contre l'image et implique même une sorte de permanente épuration qui pousse à produire (conformément aux étapes du «profil épistémologique») des concepts négatifs ou des concepts de «surobjets«, de plus en plus éloignés des intuitions de l'expérience ${ }^{9}$. C'est pourquoi la métaphore se voit en fin de compte démystifiée à un double titre: elle glisse vers le cliché, vers l'image savante, dans le champ du poétique où elle ne peut concurrencer la vivacité et la fraîcheur des images vraiment littéraires ${ }^{10}$, elle tend, d'autre part, à devenir symétriquement une congère, un foyer de résistance dans la constitution du concept en science. Bachelard, à l'image du constructivisme de la néoépistémologie a donc sanctionné le langage imagé pour ses prétentions à participer à des activités cognitives, lui réservant par contre un espace de liberté créatrice du côté de l'art et de la rêverie.

8 G. Bachelard, La formation de l' esprit scientifique, Paris, Vrin, 1967, p. 13 ss.

9 G. Bachelard, La philosophie du non, essai d' une philosophie du nouvel esprit scientifique, Paris, PUF, 1962.

10 «Alors que les métaphores ne sont souvent que des déplacements de pensée, en une volonté de mieux dire, de dire autrement, l'image, la véritable image, quand elle est vie première en imagination, quitte le monde réel pour le monde imaginé, imaginaire »: La flamme d'une chandelle, PUF, 1961, p. 2. Voir aussi J.C. Margolin, Sur les raisons d'un refus, Bachelard et la métaphore, in «Annales de l'Institut Universitaire Oriental», 1991, Naples. 


\section{2. - L'IMAGE MÉDIATRICE DU SAVOIR}

Cette attitude, qui a fini par devenir une sorte de vulgate épistémologique, n'est-elle pas, à bien des égards, sommaire, hâtive et en fin de compte injustifiée? Et surtout ne repose-t-elle pas sur des amalgames, des dérives, des pratiques caricaturales qui ne rendent pas compte de la diversité et de la complexité des situations et des pratiques? Et ne conviendrait-il pas dès lors de saisir la métaphore à l'œuvre à l'intérieur même des démarches discursives à finalité abstraite? De sorte que la rationalité ne devrait plus être invoquée comme une catégorie unique et homogène, mais faire place à des rationalités, des segments de rationalisation, qui peuvent tolérer ou même nécessiter des passages par l'image. Car les processus cognitifs les plus utilisés et validés dans les procédures des sciences, humaines en particulier, ne sont pas d'un seul tenant. Elles comportent, comme le soulignait déjà Pareto et plus récemment $\mathrm{M}$. Maffesoli ${ }^{11}$, des activités logiques, formalisables, et d'autres non-logiques, elles font appel à des démarches de mises en relation d'énoncés, mais elles doivent préalablement faire émerger ou stabiliser certains énoncés (observation, intuition, vision mentale, etc.). Autrement dit, si la métaphore peut apparaître dans la stratégie de justification d'un savoir, en servant l'activité argumentative, elle doit être à coup sûr plus active et opératoire encore dans le contexte de découverte où elle participe fondamentalement au travail d'inférences de représentations heuristiques. Il conviendrait donc bien de distinguer dans un processus théorétique des phases heuristiques, où la pensée fait des sauts, autodéveloppe des contenus en se laissant porter par des objets, des noms, des images ou des idées, et d'autres où elle enchaîne des énoncés selon des contraintes logico-formelles qu'elle s'impose délibérément. Si l'image peut sans doute à première vue être perturbatrice dans l'activité discursive rigoureuse (déduction, argumentation logique, etc.), elle n'a peut-être pas cette fonction néfaste, voire invalidante qu'on lui prête dans la genèse même des contenus de pensée. La reconnaissance de la puissance d'information des images est d'ailleurs déjà le fait des plus anciens théoriciens de la raison. On pourrait, de ce point de vue, trouver chez Platon, encore, une opération analogique exemplaire qui plaide en faveur de sa portée cognitive, le paradigme.

L'intérêt de Platon est en effet de nous faire assister, tout au long d'œuvres dialoguées, à la naissance et à la sélection de procédures de connaissance qui sont destinées à saisir l'essence des réalités empiriques. Définir ainsi une catégorie conceptuelle, le beau, le sophiste, la vertu, relève d'une série d'opérations chargées de nous émanciper de la perception des singularités et diversités empiriques pour nous amener à saisir l'unité constitutive du nom et de l'essence, qui doit seule permettre ensuite de découper en espèces réelles. Or le passage entre la perception d'exemples concrets et la saisie conceptuelle du modèle est scandé par l'élaboration d'images généralisantes. Car l'image chez Platon sert fréquemment d'intermédiaire à la connaissance entre le concret et l'abstrait ${ }^{12}$. Ainsi lorsqu'il

11 Voir G. Busino, Introduction à une Histoire de la sociologie de Pareto, Genève, Droz, 1968; et M. Maffesoli, La connaissance ordinaire, Précis de sociologie compréhensive, Librairie des Méridiens, 1985.

12 Voir V. Goldschmitt, Le paradigme dans la dialectique platonicienne, PUF, 1947. H. Bergson convoque d'ailleurs en sens inverse l'image pour passer du concept abstrait à l'intuition de l'existence unique des choses. 
s'agit de chercher l'essence du juste moral, Platon propose de déplacer l'enquête vers le juste politique, au nom d'un principe d'analogie. Car la Cité est à l'individu comme les grandes lettres le sont à de petites lettres, en elles-mêmes illisibles (République, III, 368 ss). Ce changement d'échelle d'analyse, qui repose sur un principe de proportionnalité des objets (le microcosme répète le macrocosme), fait donc, en un sens, de la Cité une métaphore de l'individu, ce qui explique pourquoi on peut parler pour la société de «corps », d' «âme» et d' «esprit» (successivement les producteurs, les défenseurs et les magistrats philosophes). La métaphorique politique trouverait donc dans l'usage de l'analogie sa raison d'être.

Mais, ce qui s'apparente encore à un raisonnement par analogie devient vraiment schématisme métaphorique lorsque Platon définit le paradigme: «Ce qui constitue un paradigme (paradeigma), c'est le fait qu'un élément, se retrouvant le même dans un groupe nouveau et bien distinct, y est exactement interprété et identifié dans les deux groupes, permet de les embrasser dans une notion unique et vraie» (Politique, 278c). Celui-ci en effet doit permettre d'établir entre des réalités différentes, des structures d'être et de connaître communes. Ainsi la technique du tissage et le métier à tisser (pris comme exemple dans le Politique) deviennent l'image métaphorique de réalités aussi hétérogènes que l'action de l'esprit sur soi, l'action de l'homme politique sur ses concitoyens, ou même que l'action du dialecticien sur les genres logiques. Dans tous les cas, l'action de croiser harmonieusement le Même et l'Autre, la ressemblance et la différence, trouve dans l'image concrète du tissage un paradigme, qui concentre et illustre une opération abstraite.

Ce pouvoir d'unification du multiple, assuré par des schèmes sensibles, peut être rapproché chez Platon de la fonction du «typos». Véritable empreinte qui imprime des signes visibles renvoyant vers les Formes modèles, le «typos » sert à rendre visible un contenu éidétique, sans pour autant l'enfermer dans un objet concret particulier qui risque à nouveau de stériliser la connaissance en l'attachant à l'accidentel et au particulier ${ }^{13}$. En ce sens, la paradigme, qui doit servir d'étape intermédiaire à la progressive saisie d'une essence, relève bien d'une typification, en laquelle l'universel et le particulier se rencontrent. Certes l'image n'est pas en tant que telle source d'intelligibilité; encore faut-il qu'elle donne lieu à une interprétation réflexive qui extraie de sa concrétude imagée les structures logiques générales qui y sont préformées. Mais il n'en reste pas moins que l'image trouve ici une légitimité et une efficacité, parce qu'elle assure une transition, une orientation de la pensée, qui resterait égarée par le réel divers et immédiat de l'expérience.

Cette leçon sera entendue, en un sens, par Max Weber, lorsqu'il assignera à la connaissance sociologique la tâche de construire pour ses objets des «types idéaux» («Ideal Type» ou «Idealbild»), qui se révèlent proches du paradigme platonicien. «Le tableau de pensée («Gedankenbild») réunit des relations et des événements déterminés de la vie historique en un cosmos non contradictoire de relations pensées... On obtient un Idealtyp en accentuant unilatéralement un ou plusieurs points de vue et en enchaînant une multitude de phénomènes donnés iso-

13 Sur le type comme hypotypose, voir Francis Goyet, De la rhétorique à la création: hypotypose, type, pathos, in J. Gayon (dir.), La rhétorique, enjeux de ses résurgences, Bruxelles, Ousia, 1998, p 46 ss. 
lément, diffus, discrets, que l'on trouve tantôt en grand nombre, tantôt en petit nombre et par endroits pas du tout, qu'on ordonne selon les précédents points de vue choisis unilatéralement, pour former un tableau de pensée homogène.» ${ }^{14}$

Il conviendrait dès lors de mieux discriminer et distribuer diverses opérations métaphorisantes, en admettant au préalable que la définition de la métaphore reste l'objet de débats pointilleux et même byzantins entre linguistes eux-mêmes. La dimension poïétique des images du langage peut, en effet, se laisser appréhender à différents niveaux, qui permettent de passer de l'image isolée (niveau moléculaire des représentations) à de véritables structures cognitives (niveau molaire), de la métaphore isolée en un mot à la métaphore filée, qui s'étend sur des phrases entières. Ce qui ne peut qu'incliner à rattacher au terme générique un éventail d'images, qu'elles obéissent à une simple comparaison sans visée synthétique, ou qu'elles apparaissent comme images génétiques, archétypiques, qui renferment à l'état virtuel une intelligiblité synthétique, comme dans le cas du symbole ou du paradigme.

\section{3. - L'ÉPISTÉMOLOGIE DE LA MÉTAPHORE FIGURATIVE}

Il n'est donc pas étonnant de voir ainsi la métaphore, libérée de son unidimensionnalité rhétorique et de sa définition réductrice, reprendre une densité, une dynamique, une fécondité abandonnées depuis longtemps. C'est pourquoi de nombreux théoriciens de la métaphore la rattachent intimement à la démarche de modélisation scientifique. Ainsi pour Max Black «Tout complexe d'implications soutenu par le sujet secondaire d'une métaphore est un modèle de prédicats attribués au sujet principal: toute métaphore est le sommet d'un modèle submergé ${ }^{15}$. De même Mary Hess décrit le démarche explicative par recours à une métaphorisation: «... le modèle déductif de l'explication devrait être modifié et complété par une vue de l'explication théorique en tant que redescription métaphorique du domaine de l'explicandum.» ${ }^{16} \mathrm{C}^{\prime}$ est pourquoi certains linguistes d'abord, certains épistémologues des sciences humaines ensuite, proposent de réévaluer la métaphore, en y voyant la marque, la trace d'une saisie originaire d'informations complexes, et non le détour gratuit par une ressemblance approximative ${ }^{17}$.

Ainsi Irène Tamba Mecz, en prenant acte de l'indistinction croissante entre comparaison et métaphore, et de l'ambiguïté du terme de métaphore, propose de faire place à une rhétorique figurative, au sens où la figure sert à désigner «tout énoncé caractérisé par la propriété sémantique d'évoquer une signification figu-

\footnotetext{
M. Weber, Essais sur la théorie de la science, Paris, Plon, 1965, p. 183.

15 M. Black, Models and metaphors, Ithaca (N.Y.), Cornell University Press, 1962, pp. 44-45.

16 M. Hesse, Models and Analogies in science, Notre Dame University Press, 1966, p. 171. F. Hallyn, à qui nous empruntons ces références, cite également à l'appui Michel Meyer, Découverte et justification en science, Klincksieck, 1979. Voir De la science comme fait rhétorique, in J. Gayon (dir.), La Rhétorique, enjeux de ses résurgences, Bruxelles, Ousia, 1998, pp. 142 ss.

17 Il conviendrait aussi de renouveler la question en montrant que la métaphore n'englobe pas seulement des substantifs, mais passe aussi par la voie du verbe. Les verbes, souvent plus imagés encore que les noms, renvoient aussi à des actions et sont donc plus proches de processus dynamiques, générateurs de pensées.
} 
rée ${ }^{18}$. A partir d'une telle réélaboration, la linguiste peut mettre à part des opérations énonciatives fondées sur un sens figuré vraiment synthétique, sans nécessité préalable de procéder à des substitutions implicites, comme le croyait la rhétorique classique. L'énoncé figuratif inaugure donc une appréhension imaginaire des choses qui rompt avec la seule visée d'ornementation. «L'énoncé figuré n'altère ni les 'choses' ni leurs 'dénominations' (les mots ne changent pas de sens), mais instaure entre elles des relations analogiques qui, littéralement, contredisent celles établies par la logique.» ${ }^{19}$ Certes en voyant dans le champ métaphorique l'entrée en scène d'un imaginaire, à son tour sous-tendu par des pulsions inconscientes, I.Tamba Mecz risque de destiner le figuré davantage à la fiction qu'à la visée cognitive. Il reste qu'elle montre que la métaphore peut échapper à son traitement dominant, asservi à un sens unique de référence.

Cette relecture du métaphorique, en linguistique, trouve d'ailleurs un appui et une illustration remarquables dans l'analyse du langage ordinaire. Sur un mode non scientifique, la pensée fait en effet déjà appel à une riche métaphorique, qui signe moins un déficit de rationalité qu'elle n'atteste la richesse de la métaphore à saisir des ensembles de significations nouées dans le quotidien, dans l'ordinaire de la vie, avant que le réel ne soit disséqué par une rationalité analytique. G. Lakoff et M. Johnson, à partir de l'analyse du langage commun, concluent ainsi à la nécessité d'arracher la métaphore au dilemme traditionnel de l'objectivisme et du subjectivisme. Sur les traces d'Aristote lui-même ${ }^{20}$, qui en souligne la vertu poïétique, il importe de reconnaître la fonction heuristique de la métaphore qui associe raison et imagination. «La métaphore est ainsi une rationalité imaginative... La métaphore est un des outils les plus importants pour essayer de comprendre partiellement ce qui ne peut être compris totalement: nos sentiments, nos expériences esthétiques, nos pratiques morales et notre conscience spirituelle. Ces efforts de l'imagination ne sont pas dénués de rationalité: puisqu'ils utilisent la métaphore, ils emploient une rationalité imaginative $»^{21}$.

C'est bien sur ce fond que l'herméneutique amplificatrice, avec à sa tête P. Ricœur, a pu faire de la métaphore «vive», un processus d'émergence du sens, renouant ainsi avec ses premières conceptions de la pensée symbolique qui lui font conclure que «l'idée d'une métaphorique initiale ruine l'opposition du propre et du figuré, de l'ordinaire et de l'étranger, de l'ordre et de la transgression. Elle suggère l'idée que l'ordre lui-même procède de la constitution métaphorique des champs sémantiques à partir desquels il y a des genres et des espèces $»^{22}$. Car le métaphorique se trouve bien ainsi entraîné dans le sillage de la symbolique, mode spécifique par lequel une pensée, tout en s'appuyant sur des contraintes syntaxiques et sémantiques de la langue, produit des agencements verbaux qui visent d'emblée un sens second, figuré, latent, qui ouvre sur une interprétation ambiguë et présuppose une sorte de transcendance du sens ( $\operatorname{Sinn}$ ) par rapport à la seule signification déno-

\section{Tamba Mecz, Le sens figuré, PUF, 1981, p. 28.}

Idem, p. 193.

20 Selon Aristote: «Les mots ordinaires transmettent seulement ce que nous savons déjà; c'est la métaphore qui peut le mieux produire quelque de nouveau ( Rhétorique, 1410b).

21 G. Lakoff et M.Johnson, Les métaphores dans la vie quotidienne, Ed.de Minuit, 1985, p. 204.

22 P. Ricœur, La métaphore vive, Seuil, 1975, p. 33. 
tative (Bedeutung), selon la distinction introduite par Frege ${ }^{23}$. La métaphore vive s'enrichit donc des vertus du symbole en tant qu'il est «le mouvement du sens primaire qui nous fait participer au sens latent et ainsi nous assimile au symbolisé sans que nous puissions dominer intellectuellement la similitude $»^{24}$.

On ne doit donc pas s'étonner que les sciences humaines, la sociologie en particulier, aient fini par prendre acte de cette nouvelle épistémologie de l'image génératrice de sens et par cautionner ou recommander une discursivité figurative, en lieu et place, ou au moins en complément, d'une rationalité formelle, conceptuelle, abstraite. En associant structure et sens, la sociologie figurative, qui s'oppose à une sociologie conceptuelle, selon $\mathrm{P}$. Tacussel, peut prétendre instaurer des analogies entre sensibilité et raison et ouvrir le chemin moins d'une explication que d'une autoréflexion éclairée par le vécu: «Les métaphores ne sont pas seulement des modèles de transposition fabriqués par le pur intellect, elles sont suggérées par l'objet social lui-même, lui-même métaphorique ou emblématique d'une figure-schème de la pensée. La construction cognitive doit donc être autoréflexive et partir à la découverte des homologies figuratives entre le social perçu et le sociologiquemen conçu.» ${ }^{25}$ La rationalité scientifique se voit donc de nos jours mise à mal, secouée dans ses assises orthodoxes. Le positivisme et le scientisme, qui ont tant fait pour l'idéalisation de la pensée homogénéisante (A. Comte annonçait, au XIX ${ }^{\mathrm{e}}$ siècle, l'avènement d'une raison positive qui devait mettre fin à la pensée métaphysique et à la pensée théologique, marquée par l'image), et qui continuent à avoir leurs prosélytes et leurs procureurs, ont connu des assauts répétés, qui aux yeux de certains caractérisent bien cette montée du non-rationnel, signe de temps post-modernes.

Il n'en reste pas moins que dans beaucoup de domaines l'épistémologie abstraite a dû faire face à une subversion, qui prend son origine dans un renversement des absolus logico-philosophiques, qui a commencé avec F. Nietzsche. Ce dernier en enracinant intimement la pensée dans les turbulences obscures du corps, en insérant la pensée dans la vie, avait déjà pu soutenir combien le concept était une production tardive et secondaire, une sorte de construction objectivante en miroir, qui se payait au prix d'une dévitalisation complète de l'objet et du sujet. C'est pourquoi, selon Nietzsche, la pensée, au contact des forces spontanées de la vie, produit d'abord des métaphores, dont les concepts sont des versions usées, fatiguées. «Qu'est-ce que la vérité? une multitude mouvante de métaphores, de métonymies, d'anthropomorphismes, bref, une somme de relations humaines qui ont été poétiquement et rhétoriquement haussées, transposées, ornées, et qui, après un long usage, semblent à un peuple, fermes, canoniales et contraignantes: les vérités sont des illusions dont on a oublié qu'elles le sont, des métaphores qui ont été usées et qui ont perdu leur force sensible, des pièces de monnaies qui ont perdu leur empreinte et qui entrent dès lors en considération non plus comme pièces de monnaie, mais comme métal.» ${ }^{26}$

\footnotetext{
23 Voir G. Frege, Ecrits logiques et philosophiques, Seuil, 1971.

24 P. Ricœur, Finitude et culpabilité, II : la symbolique du mal, p. 22.

25 P. Tacussel, Mythologie des formes sociales, Méridiens-Klincksieck, 1995, p. 31; voir aussi M. Maffesoli, La connaissance ordinaire, Librairie des méridiens, 1985.

26 F. Nietzsche, Le livre du philosophe, Garnier-Flammarion, 1991, p. 123.
} 
Un siècle plus tard, l'épistémologie subversive d'un Gilbert Durand maintient et renforce ce soupçon sur l'autonomie de la rationalité ${ }^{27}$. Dès les années 1960, l'étude des structures figuratives de l'imaginaire le conduit à distinguer trois grandes structures de composition des images (scinder, fusionner, recycler), qui pré-déterminent en fait trois types de logiques conceptuelles. Par là, la rationalité elle-même apparaît comme dérivée ou comme doublet d'une logique plus profonde qui règle des images et non seulement des concepts; la rationalité identitaire, tant revendiquée et utilisée par la raison dans les sciences, ne serait à son tour qu'une forme régionale, unilatérale, de rationalité possible. Il n'est donc pas étonnant que G. Durand assume pleinement un statut épistémique pour les symboles et les mythes et considère qu'on peut produire un savoir vrai en puisant alternativement dans le registre des mythes ou dans celui des constructions abstraites de la science ${ }^{28}$.

\section{4. - UN LOGOS VERBO-ICONIQUE?}

Qu'est-ce qui permet dès lors de fonder cette poïétique des métaphores, et la confiance qu'on peut leur faire dans les pratiques scientifiques? Faut-il seulement incriminer des réussites pratiques, ou ne faut-il pas remonter à leur condition de possibilité, c'est-à-dire montrer que la métaphore renvoie en fait à une imagination verbale et visuelle, qui participe structurellement à la construction de nos représentations, même objectives. En effet la place de la métaphore dans la rationalité scientifique ne peut se justifier seulement par la prise en compte d'un langage figuré, à côté d'un langage digital, fait de concepts définissables voire quantifiables. Car une métaphore n'est pas qu'un phénomène langagier, elle est aussi le signe qu'une pensée ne s'exprime, et peut-être ne se forme et ne se développe qu'au contact de formes spatio-temporelles et donc sensibles. De sorte que le problème de l'image dans la pensée d'abord renvoie au lien entre logos et topos, entre idéation et spatialisation, ensuite oblige à repenser le rapport entre éléments verbaux et éléments iconiques, c'est-à-dire visuels dans la représentation mentale imagée.

Trop souvent en effet, la linguistique et la rhétorique n'ont procédé à leur classification de formes et de fonctions qu'en s'appuyant sur les strictes propriétés internes du langage, oral ou écrit. Cette autonomisation de la substance langagière a conduit à minorer ou à refouler la dimension d'expérience visuelle inhérente aux faits de langage. Comme l'a fermement illustré G. Bachelard une image apparaît bien comme un complexe indissoluble d'éléments langagiers (phonétiques, sémantiques) et visuels. La «rivière» est un mot de la langue française, doté de valences dénotatives et connotatives, mais qui ne peut être séparé de la perception ou du souvenir mental d'une eau naturelle et fluide. Toute image métaphorique charrie donc avec elle une dimension verbale et une dimension iconique même si l'on peut trouver des mots sans images (dans le cas de termes techniques ou d'une

27 Voir notre étude: Pour une subversion épistémologique, in M. Maffesoli (dir.), La galaxie de l'imaginaire, dérive autour de l' œuvre de Gilbert Durand, Berg International, 1980, pp. 49. ss.

28 G. Durand, L'imaginaire, Hatier,1994; Introduction à la mythodologie, Albin Michel, 1996. 
langue étrangère inconnue) et des images déliées de mots (dans le cas du rêve $)^{29}$. La métaphore ne doit donc pas seulement être considérée comme un fait de langage mais aussi comme le témoin d'une spatialisation de la pensée. Et lorsqu'une métaphore est génétiquement première, lorsque le sens figuré repousse en arrière-plan le sens propre, bref lorsque la pensée poétise le donné, cela signifie aussi que la pensée et le langage produisent originairement des figures, qui sont bien des mises en espace, des mises en forme de contenus intellectuels. Autrement dit, la métaphore touche aux opérations élémentaires de la pensée, participe de la genèse de ses contenus, parce que la pensée a un rapport natif, structurel avec une topologie.

Cette connivence génétique entre pensée et espace est en un sens un des acquis majeurs de la philosophie contemporaine. J.-F. Lyotard, G. Deleuze, J. Derrida, M. Foucault ont chacun à leur manière mesuré l'importance de la spatialité, du lieu, du territoire, dans la conceptualisation ${ }^{30}$. Il n'est donc pas surprenant que l'image et la métaphore deviennent des modes premiers du pensé et que le travail de la pensée puisse commencer à s'actualiser comme travail par et sur des métaphores, de leur production ou de leur déconstruction. Autrement dit la topologie constitue la modalité première à travers laquelle la pensée se met en scène, se met en images. Tout logos est d'abord immanent à des images qui renvoient à des formes sensibles. Il faut donc en conclure que certaines formes spatiales donnent véritablement à penser, c'est-à-dire contiennent virtuellement des semences de vérité qu'il appartient précisément à la réflexion de dégager et d'expérimenter. Comme le montrent quelques images symboliques fondamentales, des archétypes universels, des formes prégnantes, la pensée peut y trouver des vecteurs d'intelligibilité, des types de discursivité, des logiques d'interprétation, sans concurrence possible. Cercle, ligne, croix, triangle, pyramide, œuf, corps, arbre, nœud, balance, labyrinthe, pli, fleuve, parenté, etc. ne constituent pas une encyclopédie hétéroclite d'images pédagogiques subalternes, mais une sorte de lexique et de grammaire générative de contenus de pensée, de modélisations du réel, de tropismes explicatifs du monde ${ }^{31}$. Ces agencements sensibles et spatiaux comportent dans leur propre structure sensible des nervures, des tensions, qui peuvent servir de support à des développements, des déductions, des interprétations, qui servent à rendre le monde plus intelligible. Même si ces images, métaphores, modèles peuvent être amenés à être critiqués, épurés, corrigés, ils contiennent originellement des virtualités cognitives, qui parfois deviennent des programmes d'intellection ${ }^{32}$.

Au bout du compte, du point de vue d'une théorie de la connaissance, il apparaît nécessaire d'apporter une double correction au modèle dominant: d'une part, le tissu premier dans lequel se découpent nos actes intellectifs d'explication et de compréhension est d'emblée hybride, mixte, fait d'éléments langagiers et visuels,

29 Voir notre analyse dans Philosophie des images, PUF, Thémis, 1997, pp. 23 ss.

30 Voir J.-F. Lyotard, Discours, Figure, Klincksieck, 1985. Voir aussi l'introduction de Th. Le Nain (dir.), L'image, Deleuze, Foucault, Lyotard, Vrin, 1997.

31 Nous avons amorcé quelques analyses de ce type dans L'imaginal philosophique: du cercle, de l'épée et du miroir, in B. Curatolo (dir.), L'imaginaire des philosophes, L'Harmattan,1998, pp. 9 ss.

32 Voir par exemple, Georges Poulet, Les métamorphoses du cercle, Flammarion-Champs, 1979, pour la philosophie et la littérature; et pour les sciences, Gerald Holton, L'imagination scientifique, Gallimard, 1981. 
proches de ce l'on appelle des images, avec leurs halos d'indétermination et leur strates de surdétermination affective. Cette icono-logie spontanée, loin de pouvoir être évaluée de manière binaire (positive ou négative) selon l'usage qu'on veut en faire (position de G.Bachelard), renferme à la fois des représentations fallacieuses, des préjugés erronés et des schèmes éclairants, des types dynamogènes, des noyaux d'intelligibilité éclairante. Il convient donc d'abandonner l'évaluation selon le tout ou le rien, car la métaphore n'est ni toujours stérile et trompeuse, ni toujours féconde et illuminante. Elle est à vrai dire ambivalente, plus qu'ambiguë, positivité et négativité s'y dispersant ou s'y mêlant à l'origine. Seule la pratique épistémique peut, par la méthode des essais et des erreurs, permettre de discriminer entre des obstacles et des leviers épistémologiques. L'intelligence qui se donne pour tâche d'expliquer ou de comprendre doit se comporter pragmatiquement avec ses matériaux métaphoriques, au sens large, les expérimenter, les tester, sans mépris ni idolâtrie. Nos complexes verbo-iconiques sont disponibles pour des aventures cognitives, leur destinée dépend des actes mêmes auxquels on les soumet, et des résultats qu'on veut atteindre. Les combattre systématiquement serait vain car la pensée ne peut s'immuniser contre eux, les suivre de manière échevelée, serait imprudent, car on risquerait de laisser submerger la rationalité par de l'imaginaire. La valeur d'une métaphore ne peut jamais s'apprécier a priori, elle ne peut être énoncée qu'après-coup et même parfois bien tardivement.

Ultimement, cette réhabilitation du métaphorique dans le travail conceptuel, à partir d'une théorie des facultés et des actes cognitifs, et pas seulement en fonction de leurs performances rhétoriques, conduit en fait à soutenir que la raison scientifique s'adosse ou se greffe sur une imagination. L'imagination ne saurait en effet être affectée à la seule production de fictions, de représentations irréelles, par lesquelles le sujet perturbe projectivement une connaissance du monde. Depuis Kant, l'imagination se voit reconnue deux logiques, l'une de reproduction, qui reste asservie à la représentation empirique, l'autre transcendantale et productrice de schèmes et de symboles, qui participent à la constitution, à la construction de la science et de la spéculation. Le schématisme transcendantal marque ainsi dans l'histoire des théories de la connaissance une étape décisive dans la reconnaissance d'une figuration qui ne ramène ni aux déterminations sensibles du percept ni aux déterminations abstraites du concept ${ }^{33}$. Le néo-kantisme a prolongé et confirmé cette option en montrant combien la représentation des choses, prémisses de leur intellection, se découpait dans des formes symboliques antérieures à l'intuition empirique de la chose, mais qui dotaient précisément la perception d'une prégnance symbolique ${ }^{34}$. L'épicentre de la pensée n'est donc situé ni dans les seules impressions externes (position empiriste) ni dans les seules productions internes de formes conceptuelles ou idéelles (innéisme de Platon ou Leibniz), mais dans une imagination transcendantale qui prépare le travail de la pensée,

33 Voir notre étude En quels sens peut-on parler d'imagination créatrice?, in « Revue Philosophique de l'Université de Franche-Comté», 1987, pp. 127 ss.

34 Voir les thèses de E. Cassirer dans Philosophie des formes symboliques, Ed. de Minuit, 1972, et le commentaire de O. Féron, Finitude et sensibilité dans la philosophie d'Ernst Cassirer, Kimè, 1997. 
depuis la perception de la chose jusqu'à sa subsomption sous des catégories cognitives $^{35}$.

La question de l'usage des métaphores dans les sciences humaines convoque donc un grand nombre de savoirs et d'approches et débouche sur des positions plus nuancées que les diktats des formalistes ou que le lyrisme des irrationnalistes. La métaphore, quels que soient les systèmes taxinomiques utilisés par telle ou telle linguistique, renvoie à une opération mentale complexe, qui entremêle verbal et visuel et comporte des modalités rhétoriques variées. Plutôt que de statuer normativement et définitivement, nous avons voulu faire place à un diagnostic, qui traverse une grande part de la philosophie et de l'épistémologie contemporaine. Dans ses usages langagiers comme dans les métadiscours qui les accompagnent, la métaphore se révèle non comme un instrument adjacent, contingent, de la pensée, mais plutôt comme l'effet d'une spatio-temporalisation originaire de la pensée. Car la pensée est tissée d'images langagières et optiques qui constituent son substrat hybride, mais qui s'étage selon toutes sortes de strates à fonctions cognitives spécifiques. Un schème symbolique n'a que peu de rapport avec un fantasme, un stéréotype n'a plus grand-chose à voir avec un prototype. Statuer sur le métaphorique ne peut se faire que si l'on prend préalablement soin d'explorer, de classer et de hiérarchiser cette multitude d'images. Il apparaîtra alors que dans l'imaginaire spontané se cache un «imaginal», des images ou des métaphores qui ne sont ni empiriques ni projectives, mais qui constituent de véritables noyaux, semences d'un Logos spermatique ${ }^{36}$. Mais la fécondité heuristique ou interprétative de ces formes verbo-iconiques n'est elle-même jamais assurée à l'avance. Construire une discursivité rationnelle implique que l'on déploie certaines de ces virtualités cognitives, qu'on les mette en œuvre dans des champs, des territoires herméneutiques, dont on ne pourra établir la pertinence en termes de progression de savoir et de vérité qu'après avoir atteint des résultats. Tout travail de rationalisation est habité par une imagination linguistique et visuelle, qui nous dote d'instruments tantôt bénéfiques tantôt nuisibles. Comme une arme sert à couper pour manger et survivre ou à tuer, la métaphore est vitalisante ou mortifère. Le travail de la pensée doit donc affronter cette incertitude foncière et courir l'aventure du savoir qui est toujours un grand risque. Vouloir légiférer de manière dogmatique c'est retomber dans la guerre théologique sans fin des iconoclastes et des iconodoules. La vigilance épistémologique n'a rien à voir avec quelque inquisition ou avec un esthétisme. La valeur de vérité d'une pensée scientifique se mesure à la prudence de l'usager, qui doit savoir louvoyer avec des instruments multiples et hétérogènes, et les adapter en permanence à la fin qu'elle vise. Dans ce cas, la métaphore constitue bien une donnée irréductible, une constante incompressible de la raison, mais dont la raison doit faire bon usage en cherchant toujours à exploiter ses promesses d'intelligibilité sans céder aux charmes, parfois insidieux, de ses illusions.

\section{Université de Bourgogne \\ Dijon}

35 Voir notre analyse dans l'article Philosophie et iconographie, in Encyclopédie Philosophique Universelle, Le Discours philosophique, PUF, 1998.

36 Sur la distinction entre imaginaire et imaginal, introduite par Henry Corbin, voir notre ouvrage Philosophie des images, op. cit. 